\title{
4GLS: A NEW TYPE OF FOURTH GENERATION LIGHT SOURCE FACILITY
}

\author{
M W Poole, S L Bennett, M A Bowler, N Bliss, J A Clarke, D M Dykes, R C Farrow, C Gerth, \\ D J Holder, M A MacDonald, B Muratori, H L Owen, F M Quinn, E. A. Seddon, S L Smith, \\ V P Suller and N R Thompson, Daresbury Laboratory, Warrington, UK \\ I N Ross, Rutherford Appleton Laboratory, Didcot, UK \\ B McNeil, Strathclyde University, Glasgow, UK
}

\begin{abstract}
Consideration is now being given in the UK to the provision of an advanced facility at lower energy to complement the DIAMOND x-ray light source. The proposed solution, 4GLS, is a superconducting energy recovery linac (ERL) with an output energy around 600 $\mathrm{MeV}$, delivering both $\mathrm{CW}$ beam currents up to $100 \mathrm{~mA}$ and alternatively high charge bunches for FEL applications. Production and manipulation of short electron bunches (fs) is a vital part of the source specification. In addition to beam lines from undulator sources in the ERL recovery path there will be three FELs: two will be oscillator types in the infrared and VUV respectively, and the third will be a high gain system for XUV output. The project is outlined, together with its status and the R\&D challenges posed. A funded prototype based on a $50 \mathrm{MeV}$ ERL is also described.
\end{abstract}

\section{ERL LIGHT SOURCE CONCEPT}

The origin of this project is the requirement in the UK to continue its investment in advanced radiation sources based on particle accelerators. A strategy has been developed to replace the existing national light source, the world's first dedicated $2^{\text {nd }}$ generation X-ray source (the SRS), with more advanced facilities that are optimised for specific spectral regions [1]. As a first step the $3 \mathrm{GeV} x-$ ray source DIAMOND has been funded and is now under construction [2].

For a low energy source complementary to DIAMOND it had initially been assumed that a $3^{\text {rd }}$ generation storage ring would be built, but alternative ideas emerged during 2001 [3]. In order to overcome the well known limitations of low energy storage rings below $1 \mathrm{GeV}$, and also to exploit the sub-ps bunch regime, attention turned to linacbased sources that can combine exceptionally high transverse and longitudinal brightness. Linacs have already played a major role in the development of free electron lasers (FELs) but these have usually required high peak but modest average brightness whereas a national light source will also need to deliver high average fluxes. Achieving average linac currents as high as those in $3^{\text {rd }}$ generation light source rings requires a combination of superconducting technology and energy recovery linac (ERL) principles. Such systems have now been successfully demonstrated at two laboratories [4,5], leading to a number of recent proposals to apply these results to higher energy projects generating $\mathrm{x}$-rays $[6,7]$. As an intermediate step the Cornell team have proposed a demonstrator at $100 \mathrm{MeV}$ and $100 \mathrm{~mA}$ [8]. A related series of novel light source proposals has a multi-pass configuration [9] but may omit energy recovery [10].

Once the ERL is adopted for such a spontaneous light source its superior properties also become attractive for advanced FEL development. For example the greatly enhanced peak beam currents in a linac can be exploited in a high gain FEL, by removing the mirror technology limitations of a more conventional optical cavity. Such a linac-based source then provides unique opportunities for the combination of spontaneous and stimulated (coherent) sources at a single centre: this is the unique feature of our 4GLS proposal.

\section{PROJECT OUTLINE}

The feasibility of this new light source philosophy has been studied and developed by a project team at Daresbury, together with strong strategic and scientific input from the potential user base in the UK academic community. The science case, which has emphasised the exploitation of ultra-short (fs) pulses and the extent to which multiple sources would be utilised (eg for pumpprobe experiments), was reviewed and accepted by funding agencies early in 2002. The 4GLS project concept is illustrated schematically in Fig. 1 which contains the principal components although omitting many important practical details. It has been described in more detail elsewhere [11] and will therefore only be briefly repeated here.

The $600 \mathrm{MeV}$ linac comprises a superconducting accelerating structure and one possible solution is to adapt the successful $1.3 \mathrm{GHz}$ TESLA modules [12] to the demands of a $\mathrm{CW}$ operating mode. For the purposes of 4GLS feasibility studies, including outline costings and layout considerations, a modest accelerating gradient of only $15 \mathrm{MeV} / \mathrm{m}$ has been assumed, implying a total length of about $60 \mathrm{~m}$ and $350 \mathrm{~kW}$ RF power to drive five modules. Higher gradients have been achieved at DESY 
but at much lower duty cycles; de-rating also substantially reduces the $2 \mathrm{~K}$ cryogenic burden although this may still exceed $100 \mathrm{~W}$ per cryomodule. It will also be essential greatly to enhance HOM damping and out-coupling by modifications to these structures. To achieve the target $100 \mathrm{~mA}$ the BBU instability threshold must be raised by HOM damping and by optimised transport line optics
[13]; active feedback systems will also be incorporated. Specifying a bunch length in the linac to exceed $1 \mathrm{ps}$ allows the losses to be controlled but the cryogenic plant will be rated to at least $1 \mathrm{~kW}$ at $2 \mathrm{~K}$ (ie about $1 \mathrm{MW} \mathrm{AC}$ power installation).

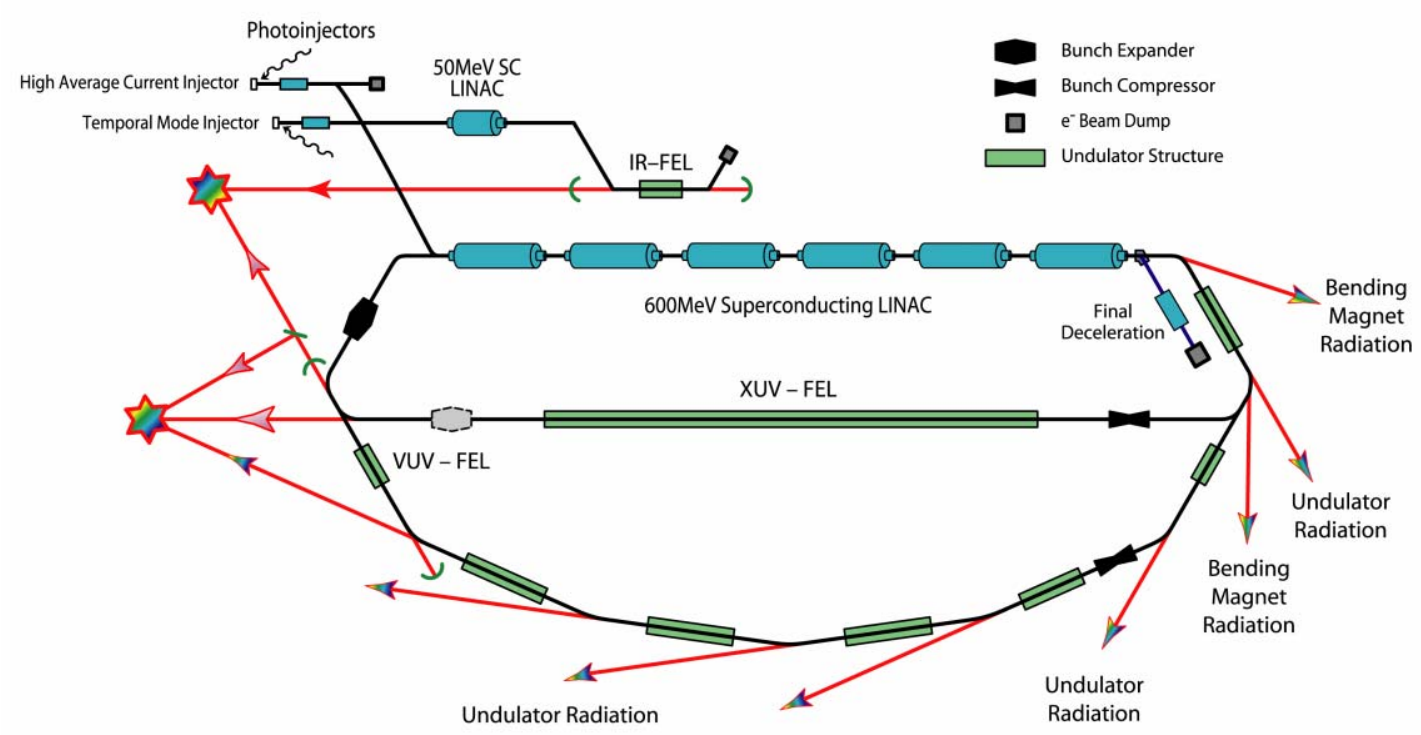

Figure 1: Schematic Layout of 4GLS Light Source Concept

Two photocathode guns are included to cover both high-average and high-peak current operating modes. A $\mathrm{CW}$ gun must be developed to deliver at least $100 \mathrm{~mA}$ in a reliable manner for a user facility. Selection of this gun will necessitate an $R \& D$ programme comparing various solutions, including DC or RF types and various cathodelaser combinations. The option of a superconducting gun will also be assessed but is unlikely to be adopted for initial stages of the project. The second gun generates the high charge per bunch needed for FEL operations: it will have a specification more similar to those being achieved in the latest linear collider and SASE FEL projects and can utilise their conclusions. This gun gives additional flexibility for production of bunch trains with a variety of pulse structures, whilst maintaining high average output for the spontaneous emission users.

Figure 1 does not detail the important electron beam transport system but it will be based on two arcs, employing tunable achromats to achieve isochronous transport over a broad energy acceptance. This is under study to determine their implications for variable $R_{56}$ control, for beam dynamics, for overall layout footprint and for technical aspects in a $600 \mathrm{MeV}$ solution. Bunch compression can be achieved both in arcs and in magnetic chicanes, but is shown schematically in Fig. 1 to indicate that fs bunch lengths will be controlled as required in local parts of the transport system. Design guidelines for arcs and chicanes are already known, in particular for minimisation of coherent synchrotron radiation (CSR) effects [14].

It should be noted that unlike other projects 4GLS needs good access to radiation from undulators (and perhaps even bends) within the arcs. Figure 1 indicates a series of beam lines feeding user stations, in some cases from fs source points, and the transport focusing optics will be locally optimised for beam dimensions at individual undulators. A cavity FEL operating in the VUV range $(3-10 \mathrm{eV})$ is also included. The electron beam can be switched into a parallel return path to feed a long undulator for a high gain XUV-FEL system to deliver 10$100 \mathrm{eV}$ output, with maximum compression immediately upstream of its entrance. Energy recovery is probably unnecessary in this low duty cycle mode having reduced average current. The infra-red FEL is supplied from an independent superconducting linac and will provide high quality, stable output over a range from perhaps 3-75 $\mu \mathrm{m}$. A feature of the 4GLS proposal is the linking of the outputs from these various sources to permit a range of pump-probe experiments to be undertaken. 


\section{ERL PROTOTYPE PROJECT}

The challenges of 4GLS are considerable and are in the areas of beam dynamics, accelerator technology, FEL physics and effective user exploitation. On 2 April 2003 it was announced that the next stage of the project evolution would be immediate funding of an Exploratory Phase. This will include construction of a smaller scale ERL Prototype (ERLP) intended to address these issues in such a test facility at Daresbury, to be completed by 2006 .

The ERLP will demonstrate energy recovery with an electron beam generated by a photocathode injector and accelerated in a superconducting linac module to at least $50 \mathrm{MeV}$. It will also incorporate an FEL test facility based on a wiggler magnet previously used in the Jefferson Laboratory IRFEL and supplied on loan as part of an international collaboration. These important physics and technology aspects will allow all of the critical 4GLS challenges to be encountered in advance of the final project construction phase.

The layout of the ERLP is now being finalised, with particular emphasis on tailoring its footprint to match an available building and infrastructure. A comparative study of Bates [15] and triple-bend achromat (TBA) arcs is underway, covering issues ranging from flexible beam dynamics through to compactness. The transport line will also include magnetic compression chicanes, to generate fs electron bunches and radiation pulses and to study associated problems (eg CSR effects, pulse synchronisation). The TBA version is shown in Fig. 2 which includes a TESLA-type linac module and the J-Lab wiggler as a reference. Injection and extraction chicanes will be needed upstream and downstream from the linac cryomodule. Start-to-end beam simulations with integrated codes have commenced and will eventually be benchmarked by the experimental data from ERLP. In parallel FEL design studies are being undertaken and will include calculation of coherent emission from the ultrashort bunches that will be available.

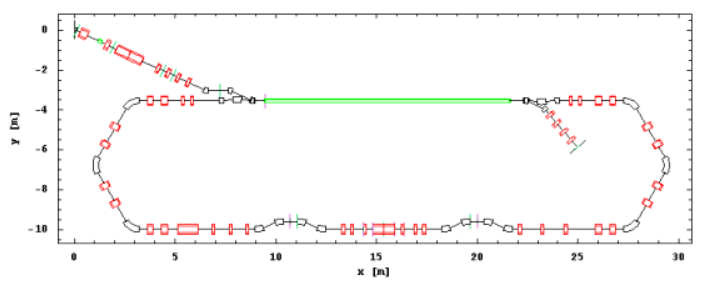

Figure 2: Preliminary ERLP Beam Transport

A photocathode gun test stand will be constructed to allow experiments to commence early in 2004 and it is likely that a DC gun based on established designs will be selected for initial experiments, in which case an RF buncher and booster will be added. It is hoped to install the linac itself and its cryogenics later that year. Attention will also be given to the advanced diagnostics necessary to make full use of the Prototype programme.

Figure 3 shows the planned layout in a converted building that already has extensive shielding and other

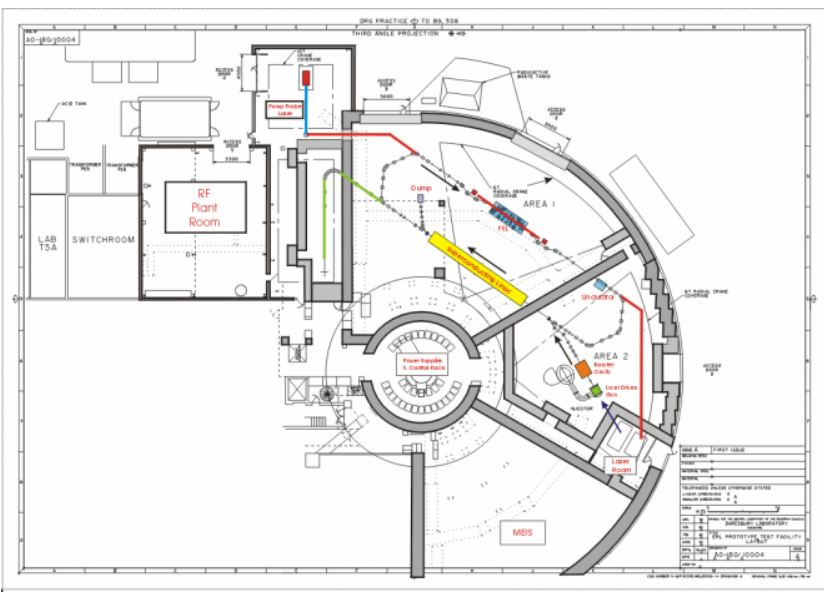

Figure 3: Possible ERLP Layout

services available. Again the TBA design is shown, but both the TBA and Bates footprints have similar dimensions and no decision has yet been made on the optimum choice.

\section{REFERENCES}

[1] M W Poole \& V P Suller, "Proposals for New UK Light Sources", EPAC'94, London

[2] R P Walker, "Progress with the Diamond Light Source Project", these Proceedings

[3] J A Clarke et al, "Prospects for a 4th Generation Light Source for the UK", PAC'01, Chicago

[4] G R Neil et al, Phys Rev Lett 84(4), 662 (2000)

[5] N Nishimori et al, "Commissioning of JAERI Energy Recovery Linac", EPAC'02, Paris

[6] I Ben-Zvi et al, "Photoinjected Energy Recovery Linac Upgrade for the National Synchrotron Light Source", PAC'01, Chicago

[7] I V Bazarov et al, "The Energy Recovery Linac (ERL) as a Driver for X-ray Producing Insertion Devices", PAC'01, Chicago

[8] I Bazarov et al, "Phase 1 Energy Recovery Linac at Cornell University", EPAC'02, Paris

[9] D A Kayran et al, "MARS: A Project of a Diffraction Limited Fourth Generation X-ray Source",APAC'98

[10] J N Corlett et al, "A Dedicated Synchrotron Light Source for Ultrafast X-ray Science”, PAC'01, Chicago

[11] M W Poole et al, "4GLS: an Advanced Multi-source Low Energy Photon Facility for the UK”, EPAC'02, Paris

[12] TESLA Technical Design Report, DESY 2001-011

[13] I V Bazarov et al, "Linac Optics for Energy Recovery Linac", PAC'01, Chicago

[14] J H Wu et al, "CSR Analysis for the Photoinjected Energy Recovery Linac and UVFEL Projects at the NSLS', PAC'01, Chicago

[15] J Flanz et al, Nucl Instr Meth A241, 325 (1985) 